\title{
The Digitalization of Lifestyle in a Digital Era: A Case Study of WeChat in China
}

\author{
Ru Ying \\ School of English for International Business, Guangdong University of Foreign Studies, Guangzhou, China \\ Email address: \\ ruying0509@gmail.com

\section{To cite this article:} \\ Ru Ying. The Digitalization of Lifestyle in a Digital Era: A Case Study of WeChat in China. International Journal of Literature and Arts. \\ Special Issue: Humanity and Science: China's Intercultural Communication with the Outside World in the New Era. \\ Vol. 8, No. 3, 2020, pp. 119-126. doi: 10.11648/j.ijla.20200803.13
}

Received: February 27, 2020; Accepted: March 10, 2020; Published: April 8, 2020

\begin{abstract}
Digitalization has been identified as one of the most significant trends that change society, business, and people's lives and has attracted academic attention from different fields. This study aims to investigate how digitalization, with the adoption of far-ranging digital technologies, has changed individuals' lifestyles in China. WeChat was taken as an example to illustrate how digital social media, a typical digital technology, penetrates every aspect of individuals' life and thus changes people's lifestyles. To obtain in-depth insights about individuals' perceptions and views towards the influence of WeChat, a semi-structured interview was conducted. Thirty respondents were interviewed, and three functionalities, namely Chats, Official Accounts and WeChat Pay, emerged as the most influential and were selected for detailed discussion. With cultural studies theories and through detailed analysis, the paper found that empowered by digital technologies, Chats has transformed individuals' communicative practices to be hyper-texted and highly interactive; Official Accounts has created a platform for individuals to follow news and express ideas, a practice that was confined to professionals only; thus, a publishing public has emerged; and WeChat Pay has revolutionized how people shop and consume and access civil service. That is, the lifestyle of individuals is digitalized in China.
\end{abstract}

Keywords: Digitalization, Lifestyle, WeChat, Chats, Official Accounts, WeChat Pay

\section{Introduction}

The last decade has seen the drastic development of the digital industry. Moreover, digitalization has attracted the attention of scholars from different academic fields. Digital techniques transform the service industry, logistics, information industry [1], consumer culture [2], and subsequently change the mass media and social media [3], which are closely related to people's daily life. Plenty of studies were conducted to investigate how media functions and what it represents in a digital era. These scholars insisted that digitalized media is a way of representing the world, and of communicating, therefore they recognize it as social and cultural processes, rather than primarily as technical ones [4, 5]. Empirically, scholars examine how people's self-representation and identities have changed on social media under the influence of digitalization $[6,7]$. While these empirical studies focused on Twitter and Facebook, Chinese scholars have conducted plenty of research on computer media platforms such as Sina Micro-blog, BBS (Bullet Board System), and Chatrooms, discussing the communicative features and how users interact in the imagined community [8-10]. However, according to the 44th China Statistical Report on Internet Development, the number of mobile phone users has reached 847 million, which brings to light the necessity of investigating the era of mobile networks in China.

WeChat is the most widely used social media application in China, a technology developed and designed for the smartphone. Though similar to Facebook in the U.S, WeChat should not be simply considered as the equivalent to Facebook. In a video report form The New York Times, WeChat was named a "super app," referred to as a "Swiss Army knife that basically does everything for you" [11]. Given its influence and prevalence, WeChat can be regarded as a typical example of digital culture in China.

Therefore, this study took WeChat as the representative of digital media in China, aiming to explore how WeChat, as a social media, changes people's life drastically. As the most 
popular social media in China, WeChat has attracted academic interests from both China and abroad. Some scholars discussed WeChat's cultural design and cultural meanings through the example of "Red Bag" or "Virtual Red Packet", and the implications of "all-in-one" feature $[12,13]$; scholars in China focus on how WeChat was applied in specific fields, including teaching, communications and how it functions [14-17]. However, few studies were conducted to examine how WeChat changes people's lifestyles from the users' perspective. In this study, the author focuses on what users do with and in relation to WeChat and aims to explain the changes with cultural studies theory, thus provides an empirical contribution to discussions that are dominated by technology scholarship. To obtain the users' views and perceptions, an interview was conducted to gain in-depth analysis of their immediate experience with WeChat. Three functionalities that arose from the interviews were chosen to be discussed in detail: the boundless and interactive messaging in Chats, the New Media represented by Official Accounts, and the pervasive WeChat Pay in mobile payment.

\section{Literature Review}

This study investigates how people's lifestyles are changed through digitalization with WeChat as an example. Toward this end, it is necessary to review studies on digitalization before reviewing the lifestyle and social media studies (WeChat is a representative of social media).

\subsection{Digitalization and Digital Culture}

Digitization and digitalization have distinctive meanings though similar in spelling. According to Gartner Glossary, digitization is "the process of changing from analog to digital form, also known as digital enablement". Drawn for the definition, it's the information that is digitized, not the processes. Digitalization is fraught with ambiguity and confusion because it's often interchangeably used with digitization but has different connotations. The definitions of digitalization are various; Gartner Glossary refers to it as "the use of digital technologies to change a business model and provide new revenue and value-producing opportunities," which focuses on changing business models. While scholars from Communications define it as "the way in which many domains of social life are restructured around digital communication and media infrastructures"[18]. Business Dictionary simply defines it as "the integration of digital technologies into everyday life by the digitization of everything that can be digitized." The latter two definitions stress social life or everyday life, i.e., how people interact. Compared digitization and digitalization, we can conclude that the latter is impossible without the former and that in digitalization it's the processes, not the information, is digitized. Therefore, digitalization means more than production and consumption of digital images, texts and sounds; it highlights the social and everyday life of individuals. To understand digitalization is essential to understand digital culture.
Digitalization has become a pervasive influence on culture. Literally, digital culture refers to a culture shaped by the emergence and use of digital technologies. But scholars attach connotations to it in specific field. Guy argued that digital technology must be imbued with social meanings in order to take root [19]. Therefore, what involves the digital transformation of society today is the diffusion of a new way of life. Since the digital technologies were invented, we have progressively discovered what they can do and at the same time we have to teach ourselves how to make use of these technologies. Out of this process, the current digital culture emerged, which is not the natural expression or extension of these technologies, but as a social selection with consequences for the same technologies. Thumim deploys in her book the term "digital culture" to invoke both the tangible and the amorphous implications of digital technology [4]. She insisted that the term 'digital culture' indicates the affordances and the constraints resulting from digital technologies shape everyday life across its multiple facets, just as electricity and print were seen as doing in previous eras. Lehdonvirta traced a brief history and termed the process of interaction between the information society and consumer society as digitalization of consumption [2]. He argued that the sites, processes, subjects as well as objects of consumption are changed by digital technologies; thus, the digitalization of consumption and consumer culture is realized. His work provides insights to understand the interaction between digital technologies and daily consumer behaviors.

Digitalization and digital culture both emphasize the influence of digital technologies on everyday life; thus, people's lifestyles are subsequently influenced and changed.

\subsection{Lifestyle and Social Media in China}

The concept of lifestyle has changed in line with societal and cultural change. Classical sociologists used the concept of lifestyle to distinguish between social classes or status groups on the basis of their cultural characteristics [20, 21]. In contemporary society, researchers attach the concept of lifestyle more importance to individually determined conditions [22, 23]. Based on what Habermas has distinguished as the three structural components of the lifeworld, namely culture, society and personality, Miegel defined lifestyle as structurally, positionally and individually determined phenomenon. As for the structural level, termed as Forms of Life, one examines differences and similarities between various countries, societies and cultures; on the positional level, termed as Ways of Living, one examines differences and similarities between large categories, classes, strata or groups within a social structure. The individual level, as the name suggests, concerns the ways in which individuals face reality and lead their lives, their relations toward other individuals, etc. in a certain social structure. It is at this level that we discuss Lifestyle [24]. Drawn on the definition and distinction, we can understand lifestyle through expressions of individuals' identity and their actions, which enables lifestyle empirically accessible.

Social media constitute an essential part of public 
communication and shape norms and rules [3] and hence is an indispensable part of everyday culture and affect people's lifestyle. A decade ago, in China, blogs and QQ, dominated the world of social media in China; then Weibo, or micro-blog, took the leading role. The latecomer WeChat overtook them with the prevalence of smart phones. Together with blogs and Weibo, WeChat is a grassroot social media. While Weibo is used as an open channel for public interaction, WeChat is a more private one for sharing and communicating with friends and colleagues. Research on social media, Twitter, Facebook and online games shows that social media promotes a more interactive and participatory communication, accompanied by multiple identity formation and new lifestyles. Being interactive, digital social media often gets altered by the efforts and needs of users, indicating the features of personalization and customization [25]. Scholars in China argue that social media bring excitement and convenience, reinforce speech right (women, youth and older adults) [26-28]. But as social media develop, contradictory ideas arise, ranging from copyright issues, personal information leakage to isolation in life, which can be demonstrated in the 2015 Kantar China Social Media Impact Report. The report showed that compared to the year 2014, the number of people doubled who held that social media made their life worse. Both the positive and negative attitudes towards social media render it a controversial issue in people's daily life and worth attention and discussion.

\section{Methodology}

As shown in the above review of literature, digitalization and social media in a digital era influence and shape people's lifestyle. And since this study takes WeChat as the representative of the social media in China, this study aims to answer the following research questions:

1) What functionalities do users of WeChat find most influential in their daily life?

2) How do these functionalities change users' lifestyle?

In order to gain a better insight into the subjective experiences of respondents' using WeChat, first, a semi-structured interview was conducted to collect respondents' views on the usage of WeChat. After the interviews were transcribed, a thematic analysis method was used to analyze the participants' views towards the features of WeChat.

\subsection{Data Collection}

Interviews allowed us to gather a wider range of perspectives than a questionnaire would have done [29]. For if we had designed a survey approach, it would have provided us with access to a larger number of participants and would have allowed us to investigate the impact of variables, such as education background, technique proficiency, and so on. However, since the purpose of the study is to focus on the major features that the users find most impressive and their subsequent views on them, I aimed to minimize imposing on the interviewees any preconceived ideas of my own.
Therefore, in this study, interviews were conducted.

The researcher collected the data of interviews. All the participants were notified the purpose of the interview, and they gave permission to the data being used in this study. In all, twenty WeChat users were interviewed. Every participant has used WeChat for at least two years. Nine of the respondents are males, and eleven are females. They are from different occupations, including engineers, civil servants, teachers, vendors, office workers and college students. The mean age is 33 , ranging from 20-62. For confidentiality, the participants were referred to by surnames, with occupation added.

The 20 participants were given an interview individually with most lasting 10-15 minutes. All interviews were conducted in Chinese. No fixed place was chosen for the interviews, since the respondents were from different occupations. Interviews for the students were conducted on campus; for office workers and civil servants, at a café; for the vendors, in the market where they work. These places are familiar to them, thus reducing pressure on them.

A total of 20 interviews were audio-recorded. Overall around 5 hours of audio recordings were collected. The researcher's notes were used as supplementary information, in case that some words or phrases were not clearly recorded due to the noisy background of certain interviews.

\subsection{Data Analysis}

First, the interviews were transcribed, and then translated into English word by word. Then the interviews were read for numerous times and a thematic analysis was conducted, which involved coding all the data before identifying and reviewing three key themes. Each theme was examined to gain an understanding of participants' perceptions and an in-depth analysis is intended. After examining the data in detail, three themes emerged, namely, Chats, Official Accounts and WeChat Pay.

\section{Findings}

\subsection{Chats}

Designed as a social media application, WeChat's most widely used functionality is Chats. As the name suggests, Chats allows users to send instant messages (IM) to WeChat contacts. The messages include text-based, or 60-s audio files, short videos and websites, which may be accompanied by images, emojis and gifs. Users may interact asynchronously, or synchronously and create messages in one or more languages. What's more, compared with traditional phone call, the mobile-data-assisted-audio-calls and video-calls enable users to make phone calls at a lower cost, regardless of the distance. New technique adds popularity to users, especially young users, who embrace the hybridity of social media. Respondents remarked chatting on WeChat as "fun, impressive and fit for group chatting".

Video calls enable users to contact without worrying the distance. Just as Sun, who lives in the U.S. remarked, "WeChat made it possible for me to see my mother the 
moment when I miss her". She interacted with her family in a WeChat Chats group, sending Virtual Red Bag (lucky money) during the Spring Festival. "I feel close to them", she concluded.

The efficiency of Chats fits into the high-paced, utility-maximizing workplace culture. Departments or work teams organize various WeChat groups to keep members in contact. In a sense, WeChat not only transforms what is communicated and how it is communicated in users' personal life but penetrates into users' working life. Chen, an engineer, said he communicated with his colleagues via two chat groups every day. They make announcements, hold online meetings and discuss job schedules. "It saves much time, and we don't bother to send e-mails."

Besides, powered by digital techniques, through Chats, users are able to share location with friends. The real-time location is regarded as the highlight of this functionality, which allows users to find each other following real-time guidance. It saves the trouble of exhaustive yet perhaps misleading description of a certain place. It's "cool and handy".

However, interviewees also complained that chatting on WeChat consumed much time and spoiled their life. Chen, the engineer, added that after work he still had to follow the chat groups in case of missing any announcements and that their colleagues now formed the habit of discussing work at night, which he thought "disturbing and exhausting". And young users expressed their worry that their friendship is "virtual", not real, because their communication is WeChat-based, and they may contact regularly, but being "more reluctant to socialize face-to-face".

\subsection{Official Accounts}

Official Accounts was another feature of WeChat that users find most important. Launched in 2012, Official Accounts allows institutions, both native and overseas business organizations, and individuals to register and upload information for publicity. It's a platform to spread information, interact with followers and promote business. WeChat users can add the accounts that interest them and become followers of the accounts. When the accounts publish news, followers will receive them instantly, then read them and give comments if they want. This functionality allows users to follow news at any time or any place, to save information for later reference and to share to more readers.

Official Accounts of functional government departments introduce a new access to their services, freeing citizens from long queues and cumbersome procedures. As two male respondents mentioned, they pay their traffic fines through the Official Accounts of traffic police department. One of the respondents shared her experience of applying for a passport on the official account of Xi' an Public Security Bureau, "quite efficient and easy to handle", she added. Two other male respondents mentioned that they followed the Traffic Police Department to check whether they violate traffic rules and pay fines if needed. Official Accounts of commercial or educational purposes cover themes of literature reading, news reports, business promotion and entertainment. Hao, 37, explained that she spent much time reading Official Accounts every day. "I follow many accounts, many. Some are about literature and technology news; some are just about entertainment gossips. They are fun to read, and they write very well."

Three of respondents registered an individual official account. Among them, Zhao (22, student) and Liu (25, student) shared their expertise on their Official Accounts. Liu uploads her movie reviews and comments on TV programs since she started her post-graduate studies in movie editing. She hopes to "interact people who share similar tastes with me, and practice for my future job". Zhao posted his articles on two themes: coffee making and traveling, neither is related to his telecommunicating major in college. He commented:

"I feel so good when people give nice comments about my photos. If they leave a comment or ask me questions, of course I'll be glad to interact with them." It is apparent that his official account not only satisfies his need to share, but his desire to be admired.

$\mathrm{Xu}$, (engineer, 25), registered his official account three years ago when he was still in college. His purpose of running his official account is simple---to earn money. Started by sharing information and selling old textbooks and lecture materials, he quickly attracted followers from his university. So far, his official account has had more than 30,000 followers. With a large subscription, his account attracts advertisers, which produces more profit. He revealed that he once made 20,000 yuan a week from commercial advertising in his account, and that's the reason why he decided to run the official account as well as keeping a full-time job related to his major in college.

Official Accounts has become a platform for both organizations and individuals to publicize or promote professional knowledge or business for free or for benefits. Users seek information or create their own accounts based on different needs, thus rendering it an individualized place.

\subsection{WeChat Pay}

After WeChat users sign up with WeChat Pay, they can get the access to this feature which enables users to shop online, order food, manage finance, etc. Users may choose to deposit money to WeChat Pay Wallet or keep WeChat hooked to a bank account with money in it. With this function, users are able to transfer money online, and also to send and receive "Red Bags" (originally means money wrapped in a red packet as gifts) electronically. According to Xinhua News, WeChat users sent billions of digital "Red Packets" as gifts or just for entertainment during the Chinese Lunar New Year. With the addition of QR Code (Quick Response Code or two-dimensional code), WeChat Pay has penetrated into every aspect of users' daily life. Each user is assigned a unique QR code which can be scanned to pay instantly and purchase quickly.

"It's quick, so quick and convenient. I don't have to prepare change money. Just scanning the QR code is enough. The customers are just waiting in line, holding their phones in 
hand." The cashier (22) in the supermarket welcomes the new payment method. And for the users, WeChat Pay saves time and trouble. The respondents all mentioned that they did not bother to take cash with them. Taking a smartphone which is equipped with WeChat enables them to dine out, buy groceries, and most importantly, take public transportations. Now subways and buses in cities of China have installed QR code scanning equipment, which allows WeChat users or Alipay (another mobile payment app in China) users to transport without buying a ticket.

Mobile payment promotes the $\mathrm{O} 2 \mathrm{O}$ (online to offline) business mode by attracting offline stores, such as cafes, restaurants, local supermarkets and so on to offer online reservation and offline delivery service. "Food Delivery" has gained popularity among office workers, students and anyone who does not want to cook or go out to eat. WeChat is in cooperation with the major food delivery apps based on the payment function. Users can also order food on the Official Accounts of the restaurants, pay through WeChat wallet and then the restaurants will deliver the food to customers.

Zhang, a college student shared her experience of ordering food online.

"I order food online nearly every day. Ur, it's convenient, fast. I can choose the time for delivery. You know, after the class, I mean, the lunch time, the canteen is always crowded."

Office workers shared similar ideas in that it saves time and offer more choices. For some young people, ordering food online is fun.

"We (my friends and I) once had a picnic in the park. We took food (with us). But suddenly we wanted to have fries. (ha-ha). So, we order chips and chickens from KFC on WeChat. And half an hour later, it was delivered to the park. So nice."

As consumption is digitalized, mobile payment creates new consumption modes. The younger generations who are used to shopping online now are provided with a new possibility.

\section{Discussion and conclusion}

\subsection{Mobile-assisted Communication in a Digital Era}

The three major functionalities of WeChat discussed above illustrated how digital technologies are modified to an individual scale. Internet is meant to connect individuals with others locally and globally; while mobile phones, empowered by current digital technologies, make it possible to fit everything in a human hand. Drawn from the respondents' experiences, smartphones and subsequent interactions via them have become an extension of the human body [19]. To be specific, with its advanced digital technologies and wide acceptance, WeChat has promoted the emergence of a diverse range of new communicative practices or habits. Unlike micro-blog users who just click "follow" to communicate, the users of WeChat communicate based on mutual agreement---both should add each other as a friend first. Compared to Tencent QQ, WeChat is connected to cellphone contacts, which renders WeChat interaction more accurate, real-time and efficient. Users have integrated WeChat into their lives and in turn WeChat has changed their habits in using social media. Users of WeChat choose to call friends through video-calls instead of making traditional phone calls, because it's cheaper and less restricted by geographic distance. They are accustomed to chatting and sharing information with individuals and groups on WeChat, thus forming various virtual communities. Instant and boundless interaction helps users to build social networks, improve work efficiency and enjoy themselves, but at the same time, users are likely to be tied to WeChat, thus spending more time interacting with others. WeChat has become part of real life and users feel unsafe without it.

Digital technologies have broadened the possibilities for interpersonal communication through social media. As in WeChat, both one-to-one and one-to-many communication are technically possible and are adopted for various reasons. WeChat, a typical digital social media, has created more interactive space. Online participatory spaces, or to be specific, participatory spaces on smartphones, represent a major part of everyday culture. Both the multi-functional Chats and Official Accounts provide a larger space for users to express themselves and interact with others. Empowered by digital technologies, participatory media have become a major part of our daily lives. According to 2017 WeChat Data Report, the number of daily sent messages via WeChat is 38 billion; the number of daily sent voice messages is 6.1 billion and that of video and voice calls is 205 million [30]. No matter what the figure masks in terms of type and kind of participation through WeChat, it is clear that the WeChat has become both a national and global phenomenon.

WeChat bridges the physical distance between distant users. For the respondents, WeChat has become part of their daily life, not just a tool for personal communication or for work. It has created new ways of being together with family members, friends and how to interact in workplaces, and through these ways, WeChat attempts to address needs and expectations (instant, interactive, participatory) that social interactions are founded on. Therefore, mobile-assisted communication is seen as a necessity rather than an option. With the help of internet communication technologies, users can potentially connect with the world, overcoming geographic distances, language differences and other barriers. Equipped with emancipating features of modern technology, internet media users not only function as audience member and consumers, but producer and organizer as well [13].

For the younger generation, online cultures extend the lived experience of physical space, and enlarge the number of opportunities for them [31]. A media platform like WeChat, has combined the need for contacts to interact with and establish a psychologically safe space with friends and family members as "virtual" company. Similar to other digital media, WeChat can be viewed as a coping mechanism for social mobility, a "comforting reminder that one is never completely alone" [31]. The efficiency and productivity of digital media benefit users, but at the same time trouble them as well. The seamless combination of professional and personal life leaves 
users less space to cultivate intimate "real" interactions. With contacts based on different communicative purposes accumulated on WeChat, users spend a large amount of time interacting with others. As respondents complained during the interview, they chat both willingly and unwillingly, "at work and after work".

\subsection{The Publishing Public}

Based on its agency, WeChat Official Accounts can be divided into four types: government organizations, media agencies, corporate accounts and individual accounts. The purposes are various: government organizations aim to spread information and promote civil services; the media agencies and corporates center on business profits, while the individual accounts are more complicated, with the purposes being personal publicity, professional knowledge dissemination as well as business profits. The wide acceptance of Official Account is a typical example of the wave of "We Media" in a digital era in China. As Peskin stated that We Media is a way how ordinary citizens, empowered by digital technologies, connect knowledge throughout the globe, contribute to and participate in their own truths, their own kind of news [32].

Digital technologies transform the media and subsequently transform how we think about ourselves. We are active producers, more than passive consumers of the media. Experience with internet media, Tencent QQ (the previous model of WeChat), Sina Weibo (micro-blog), individuals in China have been transformed into active users of media, they do not expect to be treated as anonymous invisible passive consumer; they are used to creating their own means of responding to needs and desires. Furthermore, unlike micro-blog, Official Accounts are more customized and individualized, creating a "reading community" for specific followers [33]. The accounts push news to followers after they get permission only, which proves that users play an active role in receiving information, not as passive as in previous platforms like blog or micro-blog.

We Media has offered platforms for professional practices long engaged in by the professionals only. Individuals who prefer to sell knowledge, experiences or just show themselves would create their own accounts and publish what they want to publish. This corresponds to Thumim in that online socializing networks facilitate self-representation as a strategy for increasing participation [4]. As both media agencies and individuals are enabled to express ideas about social issues, professional questions and exchange views with followers, a larger space has been created for participation and reciprocity. In traditional media, like newspapers, no matter paper versions or electronic versions, expressing views are confined to professional journalists, while on WeChat Official Accounts, both professional telecommuters and non-professional individuals have the freedom to openly express ideas and discuss issues they find meaningful; therefore, a publishing public has emerged.

\subsection{The Digitalization of Consumption}

The rapid diffusion of digital technologies and daily consumption behavior facilities the process of digitalization of consumption. The adoption of digital technologies in everyday life has exerted a profound influence on the way people consume goods and services. Consumption-related information is disseminated by groups or individuals through mobile services, and consumers are offered a variety of products for them to compare and choose, and then pay through mobile services. So, the whole process of consumption is digitalized. As consumption is digitalized, mobile payment creates new consumption modes. The younger generations who are used to shopping online now are provided with a new possibility. Users can buy through scanning a QR code in a supermarket or grocery store, without carrying cash with them. Buyers take it as "fast, convenient, saves a lot of trouble"; the sellers welcome it as well, since it saves the trouble of preparing change money for customers, thereby reducing long queues and improving efficiency.

The popularization of online shopping has transformed how people consume, and the mobile-assisted technologies increase the convenience and availability. Consumers can complete the process of consumption via a smart phone. Confronted with the convenience and availability, users may find it a habit hard to get rid of, or a part of life impossible to escape from. Digital technologies not only create new business modes and services, but also a new lifestyle in which the whole process of consumption is primarily mobile assisted. Friends, shop assistants and other human beings are totally eliminated from the process, and the consumer enters their own digital-assisted world of consumption.

Digital technologies make what used to be impossible a daily necessity. The prevalence of food delivery in China is a success of combination between online and offline world. "Dining out" once was a ritual that needs transportation time, reservation or waiting in line, but now customers just browse via their phones and choose what they prefer to eat and decide the delivered time. Modern lifestyle theoreticians argued that the processes of modernization and individualization are interwoven with the creation of lifestyles [34, 35]. Modernization is closely related to technology, while individualization accelerates the process of adopting and popularizing new technology. With the continuous introduction of new goods into society, goods that were previously considered luxuries are redefined as decencies and eventually as necessities. Digital technologies are currently undergoing such a shift. For example, the mobile phone that used to be a luxury of top executives is now an everyday necessity for Chinese citizens. As the number of smartphone users continues to rise, mobile payment users have been increasing and their habit has been further consolidated.

Apart from "food delivery", public transportation also soon adopts mobile payment. The subways, buses, even taxies are equipped with QR codes, which allows consumers to pay through scanning, without having to buy a physical card first. Users can also hail a car, make reservations, book tickets through WeChat with the help of WeChat Pay. Grabbing a phone only to go out is no longer unimaginable but has been a 
habit cultivated over months of living in China. With a Chinese bank account linked to WeChat Pay, users may shop in malls, grocery stores or subway vending machines through scanning a QR code; even street musicians get tips from their QR codes, thus city residents have gone "cashless". Though some respondents complain that digitalization "make shopping unreal" and "lead to irrational consumption", they still find it hard to resist the temptation to use it.

\section{Conclusion}

This study chose WeChat as the representative of digital social media in China, because WeChat is regarded as a "super app" via which users interact with contacts, search information, shop and entertain. Based on a semi-structured interview, Chats, Official Accounts and WeChat Pay were selected for detailed analysis. Chats transforms how users interact with contacts. With the assistance of digital technologies, users are accustomed to the efficient, instant and hyper-texted communicative practices, which have become indispensable to users' personal life and work life. Official Accounts exemplifies how media consumers are transformed to active participants in a digital era. What's more, they have the access to individualized and customized channels for publicity, where any individual can get information, promote oneself or business and report the world around them. As for the mobile payment, which penetrates into every aspect of users' life, ranging from daily shopping to civil services, users find it impossible to live without in a digital era. It not only revolutionizes business world, but also transforms how citizens live their daily life. Grabbing a phone only to communicate for life or work, pay for meals, take public transportation is now possible in China.

Because of the pervasive diffusion of smartphones in China, WeChat users have experienced a world that connects both online and off-line worlds. Since every aspect of life, such as social life, work, civil service, entertainment, etc., is assisted by digital technologies, it is easy to conclude that life at its core is a digital network connecting individuals, groups and institutions. According to the 41st China Statistical Report on Internet Development, the number of mobile Internet users in China accounted for $97.5 \%$ of the total netizen population, which indicates that mobile internet has become the cornerstone of China's digital ecosystem. Therefore, China's internet service is catered to fit in the palm of residents' hands, in terms of app functionality, variety and integration with offline world. First of all, in China, comprehensive mobile application platforms were designed to integrate social networking, information services, financial services with livelihood services, thus penetrates into every aspect of users' life. Second, immersed in the enhanced personalized scenarios, users' experiences with digital services have been upgraded. They gradually take it for granted and are accustomed to the new lifestyle. In a word, the lifestyle is digitalized.

With the detailed analysis of WeChat and respondents' views and perceptions, this study approaches how digitalization transforms people's lifestyle. It offers insights to better understand how individuals cope with digitalization. However, digitalization is a complicated and sophisticated process and it's not easy to discuss all aspects in a single article, and the selected examples only represent a small slice of people's life. Therefore, more research may be conducted to provide more interpretation of digitalization and lifestyle.

\section{Acknowledgements}

I am grateful for the careful guidance from my supervisor, and all the time and effort of all the respondents. I also thank the reviewers for their insightful suggestions regarding this work. Any remaining errors are my own.

\section{References}

[1] Toivonen, Marja; Saari, Eveliina, eds. Human-Centered Digitalization and Services. Singapore: Springer Nature Pte Ltd. 2019.

[2] Lehdonvirta, Vili. A history of the digitalization of consumer culture: From Amazon. through Pirate Bay to FarmVille. Pre-print version of a chapter appearing in Digital Virtual Consumption, ed. by J. DenegriKnott and M. Molesworth. Routledge, 2012.

[3] Dijck, José Van. The Culture of Connectivity: A Critical History of Social Media. New York: Oxford University Press, 2013.

[4] Thumim, Nancy. Self-Representation and Digital Culture. New York: Palgrave. Macmillan, 2012.

[5] Buckingham, David. Beyond Technology: Children's Learning in the Age of Digital. Culture. New York: John Wiley \& Sons, 2013.

[6] Rosengren, Karl Erik (ed.). Media Effects and Beyond: Culture, socialization and lifestyles. London: Routledge, 1994.

[7] Dobson, Amy Shields. Postfeminist Digital Cultures: femininity, social Media, and self-Representation. New York: Palgrave Macmillan, 2015.

[8] Lan, Jinsong; Yong, Peng (2000). The Psychological Influence of BBS on College Students. Contemporary Youth Research04: $20-23+15$.

[9] Zhang, Faliang (2006). Comparison and analysis of different types of virtual communities. characteristics. Research on Library Science07: 11-14+29.

[10] Xia, Yuhe (2010) The Structure and Mechanism of Micro-blog Interaction: An Empirical Study on Sina Micro-blog Journalism and Communication18 (04): 60-69+110-111.

[11] Kessel, Jonah M., Mozur, Paul (2016) August 9 How China Is Changing Your Internet Retrieved October 17, 2017, from Times Video: https://www.nytimes.com/video/technology/10000000457464 8/china-internet-wechat.html.

[12] Holmes, Kyle; Balnaves, Mark; Wang, Yini (2015) Red Bags and WeChat (Wēixìn): Online Collectivism during Massive Chinese Cultural Events Global Media Journal Volume 9 Issue I-2015. 
[13] Park, Laura J WeChat Red Bags: How International Students from China Use Social Media While Attending a Public University in California MA thesis, 2016.

[14] Fan, Wenxiang; Ma Yan; Li Kai; Qiu Bingfa (2015) A Practical Study in the Flipped Classroom Based on We Chat under Environment of Mobile Learning Open Education Research 21 (03): 90-97.

[15] Wang, Ping (2013) Analysis of Support Functions and Design Principles of Mobile Learning Based on WeChat Journal of Distance Education 31 (06): 34-41.

[16] Fang, Xiongdong; Shi Xiansheng (2013) Analysis of WeChat Communication Mechanism and Governance Issues Contemporary Communication (Journal of Communication University of China) 35 (06): 122-127.

[17] Bai, Hao; Hao, Jingjing (2013) The Application of WeChat Official Accounts in Colloge Education The Chinese Journal of ICT in Education 04: 78-81.

[18] Brennen, J S., \& Kreiss, D (2016) Digitalization In K B Jensen, R T Craig, J D Pooley, \& E W Rothenbuhler (Eds.), The International Encyclopedia of Communication Theory and Philosophy (pp 556-566) Chichester: Wiley Blackwell.

[19] Guy, Jean-Sébastien Digital technology, digital culture and the metric/nonmetric distinction Technological Forecasting \& Social Change. 145 (2019) pp 55-61.

[20] Simmel, G 'Fashion', in D N Levine (ed.) On Individuality and Social Forms Chicago: University of Chicago Press, 1971.

[21] Veblen, T The Theory of the Leisure Class An Economic Study of Institutions Harmondsworth: Penguin Books, 1979.

[22] Schudson, M Advertising, the Uneasy Persuasion: Its Dubious Impact on American Society New York: Basic Books, 1986.

[23] Turner, R. H (1978) 'The role and the person', American Journal of Sociology1: 1-23.
[24] Miegel, F Lifestyle and the Use of Media in Media Effects and Beyond, Rosengren, Karl Erik (eds), 1994 pp. 212-225.

[25] Miller, Vincent Understanding Digital Culture London: London: SAGE Publications, 2011.

[26] Zhuo, Junnan (2017) On the Rise of Females' Speech Right in Social Media networks China Newspaper Industry (06): 40-41.

[27] Wang, Yonghong (2016) Research on the Internet Public Opinion Ecology under the Influence of Mobilization and Socialization Editorial Friend (10): 53-58.

[28] Li, Qin (2017) Characteristics and social impact of the use of social media by Chinese Dama Telematics and Informatics 34 (2017) 797-810.

[29] Prior, Matthew T Interviews and Focus Groups in The Palgrave Handbook of Applied Linguistics Research Methodology London: Palgrave Macmillan, 2018.

[30] https://xw.qq.com/cmsid/20171109B0N63I00.

[31] Gregg, Melissa White Collar Intimacy in Digital Cultures and the Politics of Emotion London: Palgrave Macmillan, 2012.

[32] Peskin, Dale (2003) Introduction Bowman, Shayne \&Willis, Chris We Media: How audience are shaping the future of news and information The Media Center, pp 2-3.

[33] Pang, Shuwei (2019). The Marketing Strategies of Individual and Cultural WeChat Official Accounts. Youth Journalist (32): 92-93.

[34] Kanter, R. M., and Zablocki, B (1976). The Differentiation of Lifestyles. Annual Review of. Sociology 2 (1976): 269-98.

[35] Johansson, T. and Miegel, F. Do the Right Thing. Lifestyle and Identity in Contemporary Youth Culture. Stockholm: Almqvist \& Wiksell International, 1992. 